\title{
Article
}

\section{The shift to collaborative working and integration in the English NHS: Developing shared leadership in Primary Care Networks}

Willcocks, Stephen George and Conway, Tony

Available at http://clok.uclan.ac.uk/35946/

Willcocks, Stephen George ORCID: 0000-0002-1764-5951 and Conway, Tony (2020) The shift to collaborative working and integration in the English NHS: Developing shared leadership in Primary Care Networks. Journal of Integrated Care. ISSN 1476-9018

It is advisable to refer to the publisher's version if you intend to cite from the work. http://dx.doi.org/10.1108/IICA-07-2020-0049

For more information about UCLan's research in this area go to http://www.uclan.ac.uk/researchgroups/ and search for <name of research Group>.

For information about Research generally at UCLan please go to http://www.uclan.ac.uk/research/

All outputs in CLoK are protected by Intellectual Property Rights law, including Copyright law. Copyright, IPR and Moral Rights for the works on this site are retained by the individual authors and/or other copyright owners. Terms and conditions for use of this material are defined in the policies page. 


\section{The shift to collaborative working and integration in the English NHS: Developing shared leadership in Primary Care Networks.}

The purpose of this paper is to explore the leadership challenge of developing collaborative working with specific reference to Primary Care Networks in the English NHS, using the theoretical lens of shared leadership. This approach to leadership may be appropriate in the context of the policy shift towards collaboration and integrated care currently being pursued in the UK and in other countries. A conceptual framework is developed from extant literature, identifying the antecedents of shared leadership that help to explain how shared leadership may be developed. The framework is then used to offer insight into how shared leadership may be developed in the networks. The order of the paper is as follows: firstly, we discuss the policy background and changes in primary care in the NHS; next, we discuss shared leadership and its antecedents, and propose a conceptual framework; we then use the framework to offer a non-empirical understanding of the case of Primary Care Networks; and finally, we discuss the practical implications relating to developing shared leadership in this context.

\section{Background}

In terms of setting the context for this paper, it is important to recognize that the latest policy initiatives are part of a process of structural evolution in primary care dating back to at least 1990. This is not just a UK phenomenon: there has been considerable organizational change in primary care in other European countries , (Saltman, Rico, and Boerma, 2006). In the UK NHS, several key policy initiatives have contributed to this structural evolution. They include experiments with primary care commissioning, and the introduction of new commissioning organisations, both of which envisage a key leadership role for GPs (general practitioners). Between 1990-1997 the NHS experimented with a quasi- market in which GPs, (known as GP fundholders), were able to 'purchase' or commission a limited range of services, or take part in the Total Purchasing Pilots. Further structural evolution occurred from 1997 onwards with the creation of new primary care commissioning organisations in the form of Primary Care Groups and subsequently Primary Care Trusts, working alongside Strategic Health Authorities. In turn, these organisations were replaced in 2012 by the current commissioning organisations, known as CCGs, (clinical commissioning groups), and a new national body, NHS England, with oversight of the commissioning process and involvement in direct commissioning some services.

With regard to structural evolution specifically in general practice, there has been a gradual 'scaling up' of the traditional, single- handed, GP practice in the 1950s, to multiple- partner GP practices in the 1960s- 1970s, (Fisher, Thorlby, and Alderwick 2019). The single or individual practice has dominated in other European countries and in some of those with social insurance systems it still does, (Saltman, Rico, and Boerma, 2006). In the NHS the relatively small scale and independent nature of such practices meant that GPs were largely concerned with running a small business rather than engaging with leadership. More recently, there has been an emergence, on a voluntary basis, of new GP provider collaborations variously described as groups, clusters, federations alliances, joint ventures, multi- practice organisations, and so forth, building on pre-existing relationships of collaborating between GPs, (Pettigrew et al, 2019). There have also been experiments with collaborative working with the out-of-hours GP cooperative movement begun in 1995, (BMA /. NHS England 2019). Similarly, the 'Primary Care Homes' model introduced in 2015, bringing together GPs and other services, is said to be a precursor of the PCN, (Fisher, Thorlby, and Alderwick 2019).

These various policy initiatives and their structural implications were intended to involve GPs with leadership or governance and ensure that planning and commissioning are clinically- driven. CCGs, for example, are significant in that they are designed to be professionally-led, giving 'GPs a more influential leadership role in improving and redesigning clinical services', (Marshall, et al,2018 427). Likewise, the new GP provider collaborations have brought more involvement for GPs with leadership in primary care. They also show that collaborative working in general practice is not new, (Pettigrew, et al, 2019). In the context of this paper the 
initiatives are important in that they raise the question of how best to involve GPs in leadership, alongside their clinical role. This may be seen as part of the more general shift across the NHS towards clinical leadership. In hospitals, for example, 'hybrid' clinical leaders such as clinical directors or medical directors have been established, undertaking both a leadership and clinical role.

The above initiatives have been taken forward in the most recent examples of structural evolution in the NHS, that is, the piloting of 'new care models' designed to integrate services, and the development of integrated care systems, (NHS England, 2019). These new models of care and commissioning offer different ways of integrating health and social care, for example, unified health and social care partnerships, sustainability and transformation partnerships, and acute care or integrated community provider organisations, (NHS England 2014). The integrated community provider may be seen as a precursor to later developments in that it was intended to bring together networks of GPs and integrate with other community services, (Fisher, Thorlby, and Alderwick 2019). Building on the success of these pilots, the NHS Long Term Plan, (2019), announced the intention to 'roll- out' the development of advanced integrated care systems, aiming to have such systems in place across the country by 2021 (NHS England 2019). Significantly, the Plan introduced a system of Primary Care Networks, (PCNs), covering all GP practices within a CCG boundary but embracing a wider range of services in the local communities. PCNs will be the main vehicle to deliver 'joined -up' health and social care services in primary care based on populations of around 30-50,000 patients. They are said to represent the building blocks of the first fully-integrated system of community care since 1948, (BMA/NHS England, 2019).

Like earlier initiatives, PCNs are intended to facilitate collaborative working between GPs and with other services in primary and secondary care, (Fisher, Thorlby, and Alderwick 2019). They will be expected to influence and support relationships across the network, and work closely with both integrated care systems and their CCGs, (BMA /. NHS England 2019). Unlike earlier models, PCNs will work under a formal shared network agreement, with a shared income stream, (Fisher, Thorlby, and Alderwick, 2019). They will have to establish clear governance arrangements including agreement on decision making and accountability, and establishment of a Board/ Governing body, (NHS Confederation 2019). Each network will have a formal leader, a named accountable clinical director, to provide leadership and representation for the various GP practices. They will also need to establish multi-disciplinary teams, modelled on those that exist at secondary care level, (NHS Confederation 2019). These teams are intended to be extensions of existing independent GP partnership model teams, (NHS Confederation 2019). Such teams, known as 'extended neighbourhood teams', will include GPs, pharmacists, district nurses, physiotherapists, podiatrists, dementia workers, and staff from social care and the voluntary sector, (NHS England, 2019 14).

It can be noted that PCNs are intended to work with, but not replace the CCG. They are essentially intended to be provider -level organizations with the main commissioning role remaining with CCGs with whom they will work closely. One may argue, however, that this may bring about the potential for tension and conflict in that GPs will be expected to be involved in the governance of both organisations. In other words, they will be both commissioner and provider and this may lead to a potential conflict of interest and difficulty in making decisions. In a sense, the new arrangements could be seen as re-introducing a purchaser/provider split that was created in 1990 with GPs expected to navigate and arbitrate a complex system. Unlike in other countries with relative stability or less ambiguity in commissioning or funding arrangements, this may present a challenge for GPs and their future involvement with leadership and their relationships with other organisations.

It can also be noted at this point that the development of networks may be of wider interest, given the pursuit of integration in other countries, (Antunes and Moreira 2011). Integrated care is said to be a policy objective for healthcare systems across Europe, (Peckham, 2018). Like the UK, European countries have been concerned with the question of fragmentation and how to achieve integration and coordination in healthcare, (Saltman, Rico, and Boerma, 2006). Likewise, there are attempts 'in many European countries to channel efforts into creating improved integrated health and social care services', (Borgermans and Devroey 2017, 1). Of particular interest in the context of this paper is the fact that primary care is a key part of these initiatives, (Peckham, 
2018). In Western Europe a key development in primary care over the past 10/15 years has been the emerging role of networks, (Saltman, Rico, and Boerma, 2006). Experiments with GP networks have been conducted in France, Italy, Germany. Netherlands, and Switzerland, (Mossialos, et al, 2017; Saltman, Rico, and Boerma, 2006). Similarly, there are examples in non- European countries such as the creation of 'large independent primary medical groups' in the US, (Pettigrew, et al, 2019). Experiments with introducing networked general practice have been undertaken in New Zealand, Australia and Canada, (Fisher, Thorlby, and Alderwick 2019). In New Zealand Primary Health Organisations have been in existence since 2002. In Australia there are incentives to join a Primary Health Network; and in Canada networks of GPs are being established in several provinces, (Mossialos, et al, 2017). The policy context for these experiments may vary depending on differences in national healthcare systems such those based on taxation, like Denmark, Portugal, and the UK, or insurance- based systems such as those in Germany, Netherlands, or Bulgaria. The organization of healthcare in Europe is still considered relatively diverse, (Saltman, Rico, and Boerma, 2006). Introducing reforms in primary care may depend on the way in which each system configures its organization, funding, planning, and delivery of healthcare. The extent to which this is relatively stable and unambiguous may be important in considering such reforms. As noted, in the UK NHS there may be some ambiguity in the way in which PCNs have been configured and this presents challenges, not least for leadership.

In the next section we discuss shared leadership and its antecedents, and propose a conceptual framework.

\section{Shared leadership, antecedents, and conceptual framework}

Leader-centric approaches such as transformational leadership have been influential in healthcare since the 1980s, although such approaches have been criticized for their emphasis on the 'heroic leader' with followers seen as passive recipients in the influence process, (Lo et al, 2018). A post- heroic or post- transformational model has been proposed, with a focus on a 'nearby', or engaging style of leadership, along with the distribution of leadership across the organization, (Alimo- Metcalfe and Alban-Metcalfe 2008). This approach suggests the need to extend individual conceptualisations to embrace a shared or distributed view of leadership in which a wider range of leaders may engage, formally, or informally, with the leadership process. This wider definition of leadership is said to be appropriate in situations of change where there may be a need for 'a leadership constellation whereby the leadership role passes, informally or at different phases, between different individuals and groups, with differing bases of expertise and legitimacy at different times, (Hartley and Benington, 2010, 33). The highly fluid and changing nature of leadership that this implies is particularly relevant to the case of PCNs.

While shared leadership has been variously defined, these definitions tend to share three common characteristics, namely, that, (i) it is a lateral influence process; (ii) it is dispersed across the team, and (iii) it is emergent, (Zhu et al, 2018). With regard to the first of these, shared leadership may be defined by the way in which it engages with the influence process; it is defined as 'a dynamic interactive influence process among individuals in groups', (Pearce and Conger 2003, 1). Similarly, it is defined as 'a relational, collaborative leadership process or phenomenon involving teams or groups that mutually influence one another' (Kocolowski 2010, 24). It takes a different view of this influence process from traditional approaches such as transformational leadership where there is reliance on uni-directional influence from leader to follower, (Lo et al, 2018). A criticism of mainstream studies is that they assume that 'that leadership is an uncontested form of top down influence' (Collinson and Tourish 2015, 577).

With regard to the second characteristic, shared leadership is defined in terms of its dispersed nature, that is, who is involved in the influence process and the nature of such involvement. Unlike traditional theories, the process may be seen as a distributed activity relying on the collective personal contribution of many leaders, (Grint 2005). Thus, the process may be defined as one involving 'multiple actors who take up leadership roles both formally and informally and..[work]. collaboratively, often across organizational or professional boundaries' (Turnbull-James 2011, 4). Similarly, it is noted that shared leadership involves more than one 
leader 'stepping in and out of the leadership role when their professional expertise, particular knowledge of a client or the situation comes to the fore' (Smith et al, 2018, 9). This feature of shared leadership aligns with the third characteristic of shared leadership namely, its emergent characteristic; leaders may informally 'emerge', depending on their skills and expertise, (Pearce and Sims 2000, 120). It may be argued that all three of these characteristics are germane to the case of PCNs. Leadership in this context may be dispersed across the network- ie use many leaders - it may be based on a lateral as well as horizontal influence process, and it may allow for leaders to emerge depending on the requirements of the particular situation. Defining shared leadership in this way is particularly relevant to PCNs because leadership will be exercised not only by individual leaders such as clinical directors but, potentially, by all those in the GP communities covered by the network. Indeed, leadership is said to be a 'collective responsibility of the network'. (BMA /. NHS England 2019 97). Shared leadership- defined as a mutual, potentially emergent, influence process that is dispersed across the network- may be considered appropriate given that PCNs will be required to develop relationships and work closely with a wide range of constituencies within and outside their boundaries ensuring their commitment to the network concept. Such commitment is said to be an imperative given that it is said there is a potential risk of failure if distributed or shared models of leadership are not developed with practices in networks, (Edwards and Kumpunen 2019).

Utilising shared leadership as a theoretical framework in the case of PCNs raises the question: how does one bring about or develop shared leadership in this context? What are the conditions that might contribute to this process? There is a need to look at what influences this process, what the antecedents are from which leaders may emerge and establish which may be most conducive to such an emergence (Lord and Emrich 2001). Theoretically, it is noted that there has been limited work on determining the antecedents of shared leadership, (Serban and Roberts 2016). However, various antecedent factors have been suggested, (summarised in table 1),

\begin{tabular}{|c|c|}
\hline Author (s) & Antecedent factors \\
\hline Carson, Tesluk, and Marrone ( 2007 ) & $\begin{array}{l}\text { Internal team environment and external team } \\
\text { coaching }\end{array}$ \\
\hline Serban and Roberts ( 2016) & $\begin{array}{l}\text { Internal team environment, task cohesion, task } \\
\text { ambiguity }\end{array}$ \\
\hline Pearce and Sims (2000) & Group, task and environment characteristics \\
\hline Sweeney, Clarke, and Higgs (2019) & $\begin{array}{l}\text { Team member characteristics, composition, size, } \\
\text { internal and external environment }\end{array}$ \\
\hline Burke, Fiore, and Salas (2003) & $\begin{array}{l}\text { shared cognition, mental models, situation } \\
\text { assessment, metacognition, and attitudes }\end{array}$ \\
\hline Zhu et al, (2018) & $\begin{array}{l}\text { Formal team leader, empowering/transformational } \\
\text { leadership, and team characteristics }\end{array}$ \\
\hline Hoch (2013) & Vertical leadership and team composition/attributes \\
\hline Ulhoi and Muller (2014) & $\begin{array}{l}\text { endogenous, agency and exogenous factors, } \\
\text { including a role for formal leaders }\end{array}$ \\
\hline Fausing et al, (2015) & Empowering team leader and interdependence \\
\hline Bilal et al, (2019) & $\begin{array}{l}\text { Participative decision making, communication, and } \\
\text { power }\end{array}$ \\
\hline
\end{tabular}




\section{Table 1 Antecedent factors}

Carson, Tesluk, and Marrone (2007), for example, suggest that antecedents of shared leadership centre on: internal team environment, and, external team coaching; the former sub-divided into shared purpose, social support, and 'voice' or involvement in decision making. Similarly, Hoch (2013) identifies team member integrity- alongside vertical leadership as antecedent factors associated with innovative behaviour within the team. Fausing et al, (2015) suggest interdependence is a key antecedent factor, alongside an empowering team leader, the former representing shared goals, interaction, and interdependence. Zhu et al, (2018) also suggest the importance, alongside formal team leader, of team characteristics such as shared vision, personality compositions, integrity, and task cohesion, (Zhu et al, 2018). Other factors internal to the team include whether there is amongst team members shared vision, trust, sense of responsibility, ability to take the initiative, and interpersonal skills, (Ulhoi and Muller 2014). From a different perspective, Burke, Fiore, and Salas (2003) suggest there are four cognitive drivers that work together to facilitate shared leadership: situation assessment, (interpretation of the environment) metacognition, (monitoring/ modification of this interpretation), mental models, (cognitive structure/knowledge of the context ), and attitudes ( beliefs and dispositional attributes).

These views indicate that adopting shared leadership does not necessarily preclude the need for individual or formal leadership, and that this may be a necessary precondition to ensure the team is empowered to lead or take on shared responsibilities. Formal leaders are seen as important in creating the right culture to encourage and facilitate shared leadership amongst team members. Equally, the above views indicate some agreement relating to the importance of internal team environment alongside formal leadership as antecedent factors. These two factors do not operate in isolation and a third factor is important, recognising the role of exogenous factors ie the external environment, (Ulhoi and Muller 2014). The external environment may act as a precondition of shared leadership, for example, supportive culture, structure, power sharing, and role of line managers, (Sweeney, Clarke, and Higgs 2019). Building on this literature, a conceptual framework may be proposed, incorporating three distinct but interrelated components; (i) formal or vertical leadership; (ii) internal team environment; and (iii) external environment. For the purpose of this study internal team environment will be grouped under team- based characteristics; and individual characteristics, (Burke, Salas 2003; Hoch 2013; Pearce and Sims 2000; Zhu et al, 2018). The framework is proposed in fig 1. 


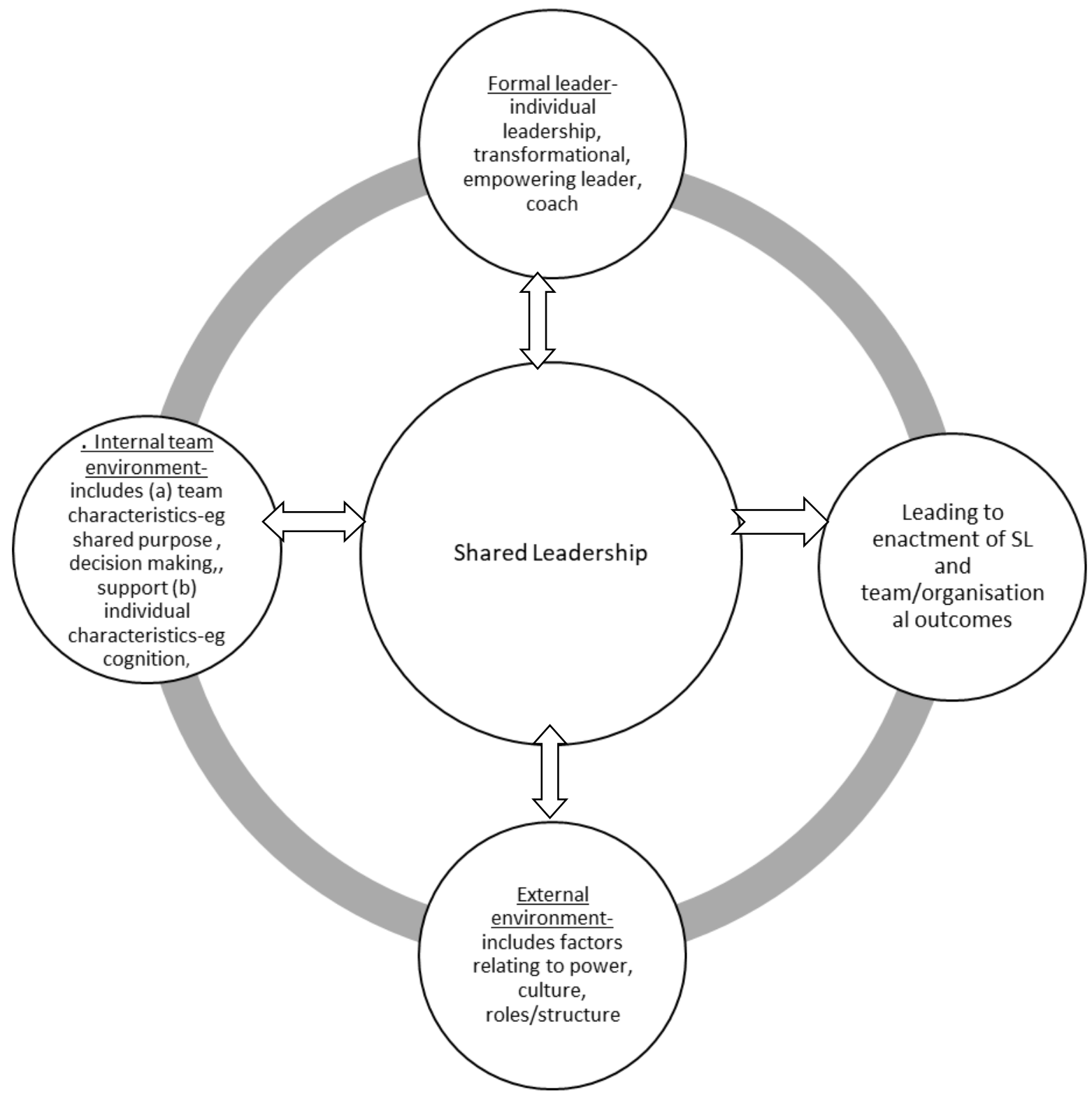

Fig. 1 conceptual framework

In the next section we utilize the conceptual framework to provide a non-empirical understanding of the development of shared leadership in the context of the new PCNs. It is acknowledged that further work will be required in testing the framework empirically.

\section{Antecedent factors for Shared leadership: the case of Primary Care networks, (PCNs).}

Fig 1 identifies a three-part categorization of antecedent factors: (i) formal leader; (ii) internal team environment; and (iii) external environment.

Formal leader

The framework suggests that individual, formal or vertical leadership may act as an antecedent of shared leadership, (Zhu et al, 2018; Hoch 2013; Ulhoi and Muller 2014). All networks have appointed an individual leader- a named clinical director- to be formally accountable for leading the network as part of the collaborative agreement, (BMA/NHS England 2019). Clinical directors will be part of a wider network including CCGs and, 
increasingly, with integrated care systems so ability to influence different constituents and develop relationships in this context may be challenging, particularly given the potentially ambiguous nature of such relationships, and constraints on their time. Evidence from other collaborative experiments indicate that good pre- existing relationships are key to these initiatives, (Pettigrew, et al, 2019). Conversely, poor relationships between practices may lead to a lack of trust and problems with decision-making, (Edwards and Kumpunen 2019). While this may be problematic for some PCNs, those with prior experience of collaborative initiatives may already have developed good relationships, trust and understanding, (Fisher, Thorlby, and Alderwick 2019). It is suggested an 'empowering', or engaging style of leadership may be relevant in this regard, (Fausing et al, 2015; , Alimo- Metcalfe and Alban-Metcalfe 2008). The ability to empower others may be seen as a prerequisite for encouraging engagement and commitment to the network. It is well known that there have been problems with GP engagement with earlier reforms. Engagement with the current reforms is vital given the fact that PCNs are faced with considerable change and have had to contend with a short timetable and rapid implementation, (Fisher, Thorlby, and Alderwick 2019). In previous collaborative initiatives in other countries engagement is said to vary depending on whether new collaborative organisations are compulsory or voluntary, (Pettigrew, et al, 2019). While the latter may help clinical engagement; compulsory initiatives 'risk clinical disengagement and even resistance', (Pettigrew, et al, 2019 53). In the case of PCNs, the arrangements are voluntary but significant resources may be lost by not getting involved.

\section{Internal team environment}

(i) team characteristics

The development of shared leadership may also depend on whether team characteristics act as antecedents of shared leadership, (Sweeney, Clarke and Higgs 2019; Hoch 2013; Zhu et al, 2018). Team-based characteristics include the ability of a team to reach a consensus on a shared purpose, goals, or vision, (Ulhoi and Muller 2014). This is said to be a key factor in developing inter-organisational collaborations, (Pettigrew, et al, 2019). Similarly, the extent to which decision- making is shared will be a key factor in influencing the development of shared leadership, (Carson, Tesluk, and Marrone 2007, Bilal, et al, 2019). Team member's professional background, training, or experience, may have an effect on their ability to reach agreement on shared purpose, or participate in shared decision- making. In some PCNs multi-disciplinary teams already existed and as such may be better placed to engage with shared leadership, (NHS Confederation, 2019). Reaching consensus and facilitating shared decision-making may be more problematic for the newly established extended neighbourhood teams given the variety of professions and care workers involved, not just in the NHS but staff from social care and the voluntary sector, (NHS England, 2019 14). Such teams may be expected to work in a complex environment, overlapping with other teams in CCGs or integrated care systems within their boundary. For GPs this may involve a transition in terms of moving from solely running a small business to involvement in a wider network arrangement. They will need to avoid working in professional silos and instead develop a sense of collective 'inter-dependence', (Fausing et al, 2015). It is noted that the transition to new ways of working away from professional silos is not guaranteed, in some cases GPs have exhibited non-collaborative behaviours in response to the introduction of PCNs, (NHS Confederation 2019).

\section{ii) individual characteristics}

Developing shared leadership in PCNs may also depend on the extent to which individual characteristics of team members act as antecedents of, or a barrier to, shared leadership. As noted, these characteristics may vary given the diversity of staff involved. Individual characteristics within the team are a key factor given that in shared leadership any member of the team may be a leader and team members will need to be able to engage in a team- based style of leadership. In other words, this implies all members of a team should potentially have leadership capabilities or characteristics, or at least have the potential to take a lead in specific areas and assume 'collective responsibility of the network'. (BMA /. NHS England 2019 97). This is consistent with the idea that shared leadership is dispersed across the team, involves shared influence and may emerge depending on circumstances. Ability to engage with leadership may already be established in some PCNs; in others there may 
be challenges. It is suggested that ability to engage may depend on team members' ability to take the initiative, their sense of responsibility, and relevant inter-personal skills, (Ulhoi and Muller 2014). Similarly, team member responsibility and trustworthiness may be an important in engaging with shared leadership, (Hoch, 2013; Zhu, et al, 2018). From a different perspective, cognition may be an important antecedent factor. Cognitive processes are said to be important for several reasons, not least, in understanding a potentially dynamic context, (Burke, Fiore, and Salas 2003). This is relevant given that individuals working in PCNs operate in a complex and potentially conflicting environment. Cognitive drivers may be important in facilitating the interpretation, monitoring/ and modifying of this environment, (Burke, Fiore, and Salas,2003). Cognitive factors may also be relevant given that individuals will need to acquire new knowledge, learn new routines and adopt unfamiliar behaviours and attitudes that are consistent with the transition to shared forms of decision making. There may be a need to make a cognitive adjustment in order to facilitate the transition to collaborative working across professional and organisational boundaries.

\section{External environment}

The framework also suggests that culture, and roles, / structures may act as antecedents of shared leadership in the networks, (Pearce and Sims 2000; Sweeney, Clarke, and Higgs 2019). Given structural evolution in primary care and the potential differences with previous collaborative initiatives such as GP commissioning or GP Federations such factors may depend on recent history and past experience. Some GP practices may be more culturally- attuned to collaborative working, the result of prior experience with earlier initiatives, whereas others may find this way of working difficult to assimilate. Equally, there may be potential differences in the way in which roles are established and enacted in the new structures given that NHS England has not been prescriptive about such roles, (NHS Confederation, 2019).. In some cases, traditional assumptions about roles may be incompatible with the ethos of shared leadership where power may be shared and where there may be both formal and informal roles. Such roles may need to be re -considered to take account of the fact that leadership will be occupied by diverse professionals at different levels, hitherto in different organisations. One of the challenges for leadership in this context is getting 'people who do not identify with being a leader to engage in leadership' (Turnbull-James 2011, 18). This has been a problem generally in getting clinicians to assume leadership roles. In hospitals this led to the creation of hybrid clinical leaders where clinicians have both a clinical and leadership role. More generally, the extent to which cultures within PCNs are supportive or resistant may be an influence on shared leadership, (Sweeney, Clarke, and Higgs 2019; Pearce and Sims 2000). Facilitating inter-professional collaborative working and sharing of knowledge and experience across differing professional cultures may be difficult, (Nzinga, McGivern, and English 2018). Differing professional cultures may exist in PCN teams. This may be problematic where such cultures dictate that clinicians deal with patients independently from one another (Smith et al., 2018). Existing professional cultures may represent a challenge for new forms of leadership.

In the next section we discuss the practical implications and development needs relating to developing shared leadership

\section{Implications in developing shared leadership}

Applying the conceptual framework has revealed the possibility of challenges to developing shared leadership in the context of PCNs. In this final section we discuss the practical implications in developing and supporting shared leadership in this context, (these are summarized in table 2). It is noted that support for leadership development in the NHS generally is not new, although in the case of primary care, the 'workforce has historically been relatively unengaged in leadership training and development', (Fisher, Thorlby, and Alderwick 2019 12). There has been a plethora of developmental initiatives in the NHS in recent years, not least those emanating from the introduction of medical leadership since the 2000s, including those associated with the Medical Leadership Competency Framework, Academy of Medical Royal Colleges, the Faculty of Medical Leadership and Management, NHS England, Health Education England, Royal College of General 
Practitioners, and NHS Leadership Academy, (Wilkie, 2012). Some of this development may be relevant to the case of PCNs, such as work- based development, fellowships, and coaching although there has been a focus on individual leadership and competency development. Individualistic approaches have been emphasized in primary care, for example, in the form of clinical leadership competency frameworks adopted by GP trainees, although these have been subject to criticism, (Moscrop, 2012). While competency approaches advocated by NHS organisations and professional bodies, may be relevant, particularly for individual leaders, development also needs to take account of the wider definition of leadership posited earlier, taking into account the fact that leadership may be dispersed across the network. It is suggested that the development needs will be different for PCNs compared to that needed in previous primary care organisations such as Primary Care Trusts, (Edwards and Kumpunen 2019). This may be related to the specific context of PCNs, for example, the fact that GPs may be involved in both CCGs and PCNs with the potential for conflicting interests. It may also be related to the relative maturity and stability of individual PCNs. This has been recognized by the development of a 'maturity matrix' with which to measure the progress of PCNs, (NHS Confederation, 2019).

\begin{tabular}{|l|}
\hline Practical implications \\
1.Individual leadership development \\
Individual support / succession planning \\
coaching \\
Skills development \\
Leadership style \\
2.Team development \\
Team skills \\
Team coaching \\
Learning together/ shared learning \\
Establishing the right team environment \\
3 External factors \\
Cultural change \\
Power sharing \\
Developing shared leadership system \\
Role definition, clarity
\end{tabular}

Table 2

Individual leadership development

Developing the leadership capability of clinical directors will be a priority for those bodies identified above such as CCGs, NHS England, Health Education England or Royal College of General Practitioners. Evidence indicates that 'credible and competent leaders' are essential to the success of collaborative initiatives, (Pettigrew, et al, 2019 54). Many clinical directors are new to this role so assuming these roles will create a particular developmental need, (NHS Confederation 2019). Support and succession planning may be needed for new directors, (Carson, Tesluk, and Marrone, 2007; Pettigrew, et al, 2019). With regard to skills development, it is noted that the challenges faced by PCNs will require 'different skills and experience from those needed in predecessor clinical commissioning groups or Primary Care Trusts, and not every clinical director will have the necessary skills and experience', (Edwards and Kumpunen 2019 10). Given the need to be able to operate in a complex environment, such skills might include networking, relationship-building, diplomacy and political 
skills to deal with potential differences that might arise across different constitutuences. Evidence from previous collaborative initiatives on the development of successful networks highlight 'the fundamental importance of clinical-managerial hybrid leaders with 'soft skills', (Pettigrew, et al, 2019 54). Similarly, evidence from CCGs suggests that at the micro level of clinical leadership 'such leaders needed to have practical knowledge and credibility among their peers to be effective', (Marshall et al, 2018). With regard to leadership style, one may argue that an empowering or, engaging, style of leadership may be appropriate, (Fausing et al, 2015, AlimoMetcalfe and Alban-Metcalfe 2008). Conversely, leaders may need 'the acumen to know when particular styles will be most effective' in a given context, in which case, a 'repertoire of styles' may be needed, (Walsh, 2016 144). It has been observed that GPs may be more likely to adopt a collaborative style of leadership than adopt 'heroic leadership styles stereotypically associated with the NHS', (Marshall et al, 2018, 429). This style may be appropriate given that the clinical directors role will involve influencing and supporting relationships, both within and outside the network, (BMA /. NHS England 2019).

\section{Team development}

In addition, team development will be a key priority, given that the new multi-disciplinary teams are intended to be a central component of the work of PCNs. Ability to engage successfully with the network may depend on the extent to which individual characteristics and team-based characteristics are given appropriate developmental support. External team -based coaching may be relevant in supporting teams, (Carson, Tesluk, and Marrone, 2007). In practical terms, such development may best be developed together, (Hartley and Benington, 2010). Likewise, unlike traditional individual -based development, there is a need to develop 'joint leadership skills together', (Walsh, 2016 144). Team skills include interpersonal skills, communication skills, listening skills, and assertiveness to enable teams to get involved with leadership. A key priority in ensuring team involvement will be to establish the right team environment in which members feel valued and are more likely to work collaboratively with ownership of team goals, (Carson, Tesluk, and Marrone 2007). Those involved in leadership and professional development such as Royal College of General Practitioners or Health Education England need to ensure that appropriate support is provided, taking into account the maturity of the PCN. It is noted that in some PCNs multi-disciplinary teams already existed and are likely to be more established, (NHS Confederation, 2019). In the case of the primary care homes initiative or integrated community providers, experience indicated that 'some will already have strong cross- practice relationships, trust and understanding-all necessary foundations for successful collaboration', (Fisher, Thorlby, and Alderwick 2019 14). The importance of these foundations is supported by previous research, for example, research in general practice found that collaborative working was most successful where it was underpinned by a clear focus, good relationships, trust, and support; whereas ' a lack of clarity of purpose or engagement or overoptimistic expectations', were factors working against successful collaboration, (Kings Fund 2019, 2). Similarly, evidence from other general practice initiatives, identified trust and good working relationships between GPs as important success factors in collaborative initiatives, (Pettigrew, et al, 2019).

\section{External factors}

Finally, there are broader implications for those involved with PCNs, in particular, CCGs and NHS England, in terms of supporting culture change, establishing new roles and governance structures, and minimizing the effects of potential ambiguity in the relationship between PCNs and CCGs. These are said to be key areas that will need support, (NHS Confederation, 2019). These aspects cannot be taken for granted as the history of structural evolution in primary care has revealed differences in take up and involvement with various policy initiatives and problems of clinical engagement. It has been noted from experience with other initiatives that clinicians are not always involved or engaged with 'clinically -led configurations', (Iles and Ahluwalia 2015, 56). Engaging with leadership roles in governance structures may require clarification, given that such roles may be unfamiliar. For example, in the case of the clinical director there may a need to clarify how this role interacts with the constituent members of the network, and with other organisations such as CCG or integrated care system. A study of CCGs suggests that there may be three levels of leadership: (i) a macro- level, formal strategic role; (ii) a meso level programme leadership; and (iii) a micro-level where clinical leaders were 
involved in an operational role, (Marshall et al, 2018). This is likely to be required in the case of PCNs, and support will be required for creating a governance system not only at the macro-level, occupied by the clinical director but at meso and particularly micro levels, the domain of GPs and other professionals. This will require clarity and the creation of 'clear roles for each of the different levels of the local system', (Edwards and Kumpunen 2019,8). On the other hand, some acceptance may be needed that leadership roles will be fluid, (Burke, Fiore, and Salas, in Pearce and Conger, 2003). Those involved in supporting the development of PCNs such as NHS England and CCGs will also need to ensure attention is given not just to roles and structure but to the political and cultural aspects of the change. Lessons from provider experience on engaging with PCNs suggest that culture is a strategic priority, (NHS Confederation, 2019). It can be noted that moving networks along the developmental continuum will be achieved as much by cultural as formal, structural means, although it has been argued that both culture and structure are antecedents of shared leadership, (Sweeney, Clarke, and Higgs 2019). It can also be noted that the political environment is important in supporting the development of shared leadership. In the case of CCGs, research has suggested that the political environment of CCGs was not entirely supportive of clinical leadership, (Marshall et al, 2018).

\section{Conclusion}

This paper has suggested that shared leadership may be an appropriate theoretical lens with which to explore the development of leadership in the case of PCNs. It has proposed a conceptual framework that might be used to identify the antecedent factors that contribute to understanding this development It has been noted that theoretical and empirical work in this regard so far has been limited, (Serban and Roberts 2016; Fausing et al, 2015).

An understanding of such factors may be relevant to the new PCNs given their central role in developing collaborative working in an integrated system of care. The extent to which new PCNs are able to embrace collaborative working, and facilitate integration of services across the network community, may be dependent on the extent to which such factors are supportive of the emergence of shared leadership. Based on extant literature, the paper has proposed that these factors may be categorized as (i) the individual leader; (ii) the internal team environment; and (iii) the external environment in which PCNs operate. An overriding factor will be the fact that shared leadership ethos and mindset needs to be nurtured in a context which, in the past, has experienced problems with clinical engagement. It is noted that developmental implications of this may also depend on the level of organisational maturity of each PCN and the extent to which they have had prior experience of collaborative working.

\section{References}

Alimo- Metcalfe, B and Alban-Metcalfe, J. (2008) Engaging leaders-Engaging for success, London: CIPD, September, 1-38

Antunes, V and Moreira, J, P. (2011), Approaches to developing integrated care in Europe: a systematic literature review, Journal of Management \& Marketing in Healthcare, 4:2, 129-135

Bilal, A. R, Fatima, T, and M, K Imran, ( 2019), Does shared leadership fosters taking charge behaviours? A post-heroic leadership perspective in the public sector higher educational institutes, International Journal of Public Leadership 153 137-154

BMA/NHS England, (2019), Investment and Evolution: A five year framework for GP contract reform to implement The NHS Long Term Plan, London, BMA / NHS England 31 January

Borgermans, L. and Devroey, D. ( 2017), A Policy Guide on Integrated Care (PGIC): Lessons Learned from EU Project INTEGRATE and Beyond. International Journal of Integrated Care, 17(4): 8, pp. 1-12 
Burke, C. S., Fiore, S, M. and Salas. E. (2003), 'The Role of Shared Cognition in enabling shared leadership and team adaptability', in C. L Pearce, and J, A.Conger, (eds.), Shared leadership- Reframing the Hows and Whys of leadership, London: Sage.

Carson, J, B, Tesluk, P, E. and J, A. Marrone, (2007), “ Shared leadership in teams: an investigation of antecedent conditions and performance". Academy of Management Journal, 50,5, 1217-1234

Collinson, D and Tourish, D. (2015), "Teaching leadership critically: New directions for leadership pedagogy", Academy of Management Learning and Education, 14, 4, 576-594

Edwards, N and S, Kumpunen, (2019) Primary Care Networks: a pre mortem to identify potential risks, London, Nuffield Trust

Fausing, M, S; Joensson, T, S; Lewandowski, J; and M, Bligh, (2015) "Antecedents of shared leadership and interdependence", Leadership and Organisation Development Journal 36 3271-291

Fisher, R, Thorlby, R and H Alderwick, (2019) "Understanding primary care networks Context, benefits and risks" London, The Health Foundation, July 1-21

Fu, L, and Z, Liu, (2018) "Distributed leadership in organisations: an investigation of antecedent conditions", Chinese Management Studies, 124 682-670

Grint, K. (2005), Leadership: Limits and Possibilities, Basingstoke Hampshire: Palgrave Macmillan.

Hartley, J and Benington, J (2010) Leadership for Healthcare, Bristol,The Policy Press

Ham, C. (2018),Making sense of integrated care systems, integrated care partnerships and accountable care organisations in the NHS, London: Kings Fund.

Hoch, J, E. (2013), Shared leadership and innovation: the role of vertical leadership and employee integrity, Journal of Business Psychology, 28 159-174

Iles, V, and and S,Ahluwalia, (2015) "Clinically led or clinically driven?” British Journal of General Practice, January55-57

Kings Fund, (2019), Primary care networks explained, London: Kings Fund

Kocolowski, M D. (2010), “Shared leadership: is it time for a change?”, Emerging leadership Journeys, $3,1,: 22-32$

Lo, D, J, McKimm, and A. Till, (2018) Transformational leadership; is this still relevant to clinical leaders? British Journal of Hospital Medicine, June Vol 79 No 6, 344-347

Lord, RG and Emrich, C G, ( 2001)Thinking outside the box by looking inside the box: Extending the cognitive revolution in leadership research, Leadership Quarterly, 11,4, 551-579

Marshall, M, Holti, R, Hartley, J Matharu, T, and J,Storey, ( 2018), ,GP leadership in clinical commissioning groups: a qualitative multi-case study approach across England," British Journal of General Practice, June 427432

Moscrop, A (2012) Clinical leadership-individual advancement, political authority, and a lack of direction? British Journal of General Practice May384-386 
Mossialos, E, Djordjevic, A, Osborn, R, and Sarnak, D (Eds), (2017),International profiles of health systems, New York, The Commonwealth Fund, 31 May

NHS Confederation, (2019) Primary Care Networks: A quiet revolution, London NHS Confedration and NHS Providers

NHS England 2014),NHS Five Year Forward View, London, NHS England

NHS England, (2017), Next steps on the NHS five year forward view, London: NHS England

NHS England, (2019), The NHS Long Term Plan, 7 January, London: NHS England

Nzinga, J, McGivern, G, and English, M. (2018), "Examining clinical leadership in Kenyan public hospitals through the distributed leadership lens, Health Policy and Planning,33,2, ii27-ii34

Pearce, C L and Conger, J A, (2003), 'All those years ago: the historical underpinnings of shared leadership', in C, L.Pearce, and J, A.Conger, (eds.), Shared leadership- Reframing the Hows and Whys of leadership, London: Sage

Pearce, C, L. and H. P,Sims, (2000), " Shared leadership: towards a multi-level theory of leadership", Team Development, 7, 115-139

Peckham, S. (2018), Developing an integrated primary care workforce, European Journal of Public Health, 28 (4) November

Pettigrew, L, M, Kumpunen, S, K, Rosen, R, Posaner, R, and Mays, N (2019), Lessons for large scale general practice provider organisatiions in England from other inter-organisational healthcare collaborations, Health Policy, 123 51-61

Rawlings D, ( 2000), “Collaborative leadership teams: Oxymoron or new paradigm”? Consulting Psychology Journal: Practice and Research, 52,1, 36-48

Saltman, R, B. Rico, A. and Boerma, W. (Eds) (2006), Primary Care in the Drivers seat, European Observatory on Health Systems, OU Press

Serban A and Roberts, A, J. B. (2016), "Exploring antecedents and outcomes of shared leadership in a creative context: a mixed methods approach”, The Leadership Quarterly, 27, 181-199

Smith, T, S.Fowler-Davis, S. Nancarrow, S, M, B. Ariss, and P.Enderby, (2018), "Leadership in interprofessional health and social care teams: a literature review”, Leadership in Health Services, 31,4, 452-467

Sweeney, A, Clarke, N, and M, Higgs, (2019) "Shared leadership in commercial organisations: a systematic review of definitions, theoretical frameworks and organisational outcomes", International Journal of Management Reviews, 21 115-136

Turnbull-James, K. (2011), Leadership in context: lessons from new leadership theory and current leadership development practice, London: Kings Fund, Commission on Leadership and Management in the NHS

Ulhoi, J, P. and S, Muller, (2014) "Mapping the landscape of shared leadership: a review and synthesis," Internatiional Journal of Leadership Studies, 82 66-87 
Walsh, K, (2016), Clinical leadership education: the future, British Journal of General Practice, March, 144

Wilkie, V. (2012). Leadership and management for all doctors, British Journal of General Practice, May 230231

Zhu, J, Liao, Z, Yam, K, C, and R, E, Johnson, (2018) "Shared leadership: a state of the art review and future research agenda, Journal of Organisational Behaviour, 39 834-852 
